\title{
Net-baryon number fluctuations with the hadron resonance gas model using Tsallis distribution
}

\author{
D. K. Mishra ${ }^{1}$, P. Garg ${ }^{2}$, P. K. Netrakanti ${ }^{1}$ and A. K. Mohanty ${ }^{1}$ \\ ${ }^{1}$ Nuclear Physics Division, Bhabha Atomic Research Center, Mumbai 400085, India \\ 2 Department of Physics, Banaras Hindu University, Varanasi 221005, India \\ E-mail: dkmishra@rcf.rhic.bnl.gov
}

\begin{abstract}
We explore a hadron resonance gas model using Tsallis non-extensive distribution to study the energy dependence of the product of the moments, $S \sigma$ and $\kappa \sigma^{2}$ of net-proton multiplicity distributions of published STAR data in $\mathrm{Au}+\mathrm{Au}$ collisions at relativistic heavy-ion collider (RHIC) energies. While excellent agreements are found between model predictions and measurements of $S \sigma$ and $\kappa \sigma^{2}$ of most peripheral collisions and $S \sigma$ of most central collisions, the $\kappa \sigma^{2}$ for most central collisions deviates significantly from the predictions particularly at $\sqrt{s_{N N}}=19.6 \mathrm{GeV}$ and $27 \mathrm{GeV}$. This could be an indication of the presence of dynamical fluctuations, which are not contained in the HRG-Tsallis model.
\end{abstract}

PACS numbers: 25.75.Gz,12.38.Mh,21.65.Qr,25.75.-q,25.75.Nq 
Net-baryon number fluctuations with the hadron resonance gas model using Tsallis distribution2

\section{Introduction}

The energy dependence of the moments (mean $M$, variance $\sigma$, skewness $S$ and kurtosis $\kappa)$ and their products $\left(S \sigma\right.$ and $\left.\kappa \sigma^{2}\right)$ for net-proton multiplicity distribution at RHIC energies are reported by STAR collaboration [1]. The product of the moments $S \sigma$ and $\kappa \sigma^{2}$ can be linked to the ratios of susceptibilities $(\chi)$ associated with the baryon number conservation [2, 3, 4]. For example, the product $S \sigma$ can be written as the ratio of third order $\left(\chi_{B}^{3}\right)$ to second order $\left(\chi_{B}^{2}\right)$ and the product $\kappa \sigma^{2}$ as the ratio of fourth order $\left(\chi_{B}^{4}\right)$ to second order $\left(\chi_{B}^{2}\right)$ baryon susceptibilities. The recent STAR measurements of $S \sigma$ and $\kappa \sigma^{2}$ show significant deviations from the predictions of the Skellam distribution (where $\kappa \sigma^{2}$ should be unity) at all energies, indicating the presence of large non-statistical fluctuations [5]. The particle production in heavy ion collisions at relativistic energies are well described in terms of the hadron resonance gas (HRG) model where fermions and bosons follow Fermi-Dirac (FD) and Bose-Einstein (BE) distributions, respectively [6, 7]. The success of HRG model would mean that the created thermal system which might have gone through a possible phase transition, has (nearly) equilibrated both thermally and chemically at freeze-out. It is believed that if the thermal system has retained some memory of the phase transition with finite correlation length at freeze-out, it must be reflected in the higher moments of the conserved quantities [7, 8, 9]. Although it is not so obvious in the study of thermal abundance of the individual species. Therefore, the study of fluctuations in various conserved quantities such as: net-charge, netstrangeness and net-baryon number through the higher moments using HRG model is expected to provide a baseline to observe the deviation in experimental observables. The deviations of experimental data from these model studies may indicate the presence of non-statistical fluctuations, if any.

The HRG model in Boltzmann approximation follows an exponential behavior of particle production corresponding to Boltzmann-Gibbs (BG) statistics. Recently, it has been shown that particle production both in heavy ion and proton-proton collisions at RHIC and large hadron collider (LHC) energies can be described successfully using a power law distribution at high transverse momentum rather than using the exponential one [10, 11, 12]. Therefore, the Tsallis distribution function is being used for particle production with non-extensive parameter $q$ such that it approaches Boltzmann distribution in the limit $q \rightarrow 1$. In the context of particle production in heavy ion collisions, Tsallis distribution has been interpreted as the superposition of Boltzmann distributions with different temperatures [13. Such a situation can occur when the hot and dense medium created in high-energy nuclear collisions is not homogeneous in temperature but fluctuates from point to point around some equilibrium value $T_{f}$ [14, 15]. The temperature fluctuation which exists in small part of the phase space with respect to the whole is another source of non-statistical fluctuation. This is different from the statistical fluctuations measured on an event by event basis and should be properly accounted in the model. In general, Tsallis non-extensive statistics is supposed to include situations characterized by long range interactions, long range microscopic 
memory and space time fractal structure of the process [16, 17]. There could also be other non-statistical fluctuations of dynamical nature associated with phase transition where the correlation length diverges at the critical point [18]. It may so happen that either due to finite size effect or if the freeze-out occurs at a temperature far away from the critical point, the strength of the dynamical fluctuations becomes progressively weaker. However, these dynamical fluctuations can still be studied through higher moments which diverge faster than the lower moment like the variance [19].

The success in understanding of non-equilibrated complex systems using Tsallis statistics [16, 20] has motivated many phenomenological studies in understanding the particle production in elementary $p+p$ collisions at RHIC [21, 22] and LHC [23, 24]. For heavy ion collisions, where the system formed is not a simple superposition of many $p+p$ collisions, Tsallis functional forms are successfully applied to describe the transverse momentum and rapidity distribution of the produced particles at different collision centralities and center of mass energies [10, 25, 26]. The non-extensive parameter $q$ characterizes the degree of non-equilibrium in the system. It indicates the deviations in the particle production mechanism from simple BG statistics and could give insight about the intrinsic fluctuations during hadronization process in the heavy-ion collisions [13].

In this paper, for the first time, we consider a hadron resonance gas model using Tsallis non-extensive distribution (HRG-Tsallis) to study non-statistical fluctuations of net-baryon (or net-proton) production in $\mathrm{Au}+\mathrm{Au}$ collisions at RHIC energies. In the limit of $q \rightarrow 1$, we reproduce the HRG results. We show that the HRG-Tsallis model with a temperature dependent non-extensive $q$ parameter can explain the energy dependence of $S \sigma$ and $\kappa \sigma^{2}$ for most peripheral $(70-80 \%)$ collisions, but fail to explain the same for central $(0-5 \%)$ collisions. However, the energy dependence of $\kappa \sigma^{2}$ from experimental data for central collision deviates significantly from the HRG-Tsallis model predictions particularly at energies $19.6 \mathrm{GeV}$ and $27 \mathrm{GeV}$. We argue that the predictions of HRG-Tsallis characterized by a temperature dependent $q$ parameter should be taken as the baseline to study (experimentally) fluctuations of dynamical origin if any, which are still not contained in the Tsallis non-extensive thermodynamics.

The paper is organized as follows. In Section 2, we will discuss the HRG model with Tsallis distribution used in this study and interpretation of generalized susceptibility in terms of non-extensive parameter $q$. Section 3 describes the temperature dependence of $q$ parameter. In Section 4, comparison of the results for $\chi^{(3)} / \chi^{(2)}$ and $\chi^{(4)} / \chi^{(2)}$ between STAR experimental data for net-protons and HRG-Tsallis predictions for the net-baryon distributions for the most peripheral (70-80)\% and the most central (0$5) \% \mathrm{Au}+\mathrm{Au}$ collisions. Finally in Section 5, we summarize our findings and mention about the implications of this work to the current experimental measurements of higher moments in high energy heavy-ion collisions. 
Net-baryon number fluctuations with the hadron resonance gas model using Tsallis distribution4

\section{HRG-Tsallis Model}

The Tsallis form of FD and BE distribution can be written as [27],

$$
f=\frac{1}{\exp _{q} \frac{(E-\mu)}{T} \pm 1}
$$

where ' \pm ' signs are used for fermions and bosons, respectively and $\exp _{q}(x)$ is given by,

$$
\exp _{q}(x)=\left\{\begin{array}{lll}
{[1+(q-1) x]^{1 /(q-1)}} & \text { if } \quad x>0 \\
{[1+(1-q) x]^{1 /(1-q)}} & \text { if } \quad x \leq 0
\end{array}\right.
$$

where $x=(E-\mu) / T$. The above distribution approaches standard FD and BE distributions in the limit $q \rightarrow 1$. Using the above non-extensive distribution function Eq. 1, the average number density can be written as,

$$
n_{q}=\sum_{i} g_{i} X_{i} \int \frac{d^{3} k}{(2 \pi)^{3}} f_{i}^{q}\left(E_{i}, T_{f}, \mu_{i}\right),
$$

where $T_{f}$ is the chemical freeze-out temperature, $\mu_{i}$ is the chemical potential and $g_{i}$ is the degeneracy factor of the $i^{\text {th }}$ particle. The total chemical potential $\mu_{i}=B_{i} \mu_{B}+Q_{i} \mu_{Q}$ $+S_{i} \mu_{S}$, where $B_{i}, Q_{i}$ and $S_{i}$ are the baryon, electric charge and strangeness number of the $i^{t h}$ particle, with corresponding chemical potentials $\mu_{B}, \mu_{Q}$ and $\mu_{S}$, respectively. The term $X_{i}$ represents either $B, Q$ or $S$ of the $i^{t h}$ particle depending on whether the computed $n_{q}$ represents baryon density, electric charge density or strangeness density, respectively. The factor $d^{3} k$ can be expressed in terms of transverse momentum $\left(p_{T}\right)$, pseudo-rapidity $(\eta)$ and azimuthal angle $(\phi)$ as, $d^{3} k=p_{T} \sqrt{p_{T}^{2}+m^{2}} \cosh \eta d p_{T} d \eta d \phi$ and

energy $E$ is expressed as, $E=\sqrt{p_{T}^{2}+m^{2}} \cosh \eta$. Note that the exponent $q$ in $f_{i}$ has been introduced as a constraint for thermodynamical consistency [27]. Since the average density and the pressure $P$ are shown to be thermodynamically consistent i.e. $n_{q}=\frac{\partial P_{q}}{\partial \mu}$, we can now define generalized susceptibilities as,

$$
\chi_{q}^{n}=\left.\frac{\partial^{n}\left[P_{q}\left(T_{f}, \mu\right)\right]}{\partial \mu^{n}}\right|_{T}=\frac{\partial^{n-1}\left[n_{q}\left(T_{f}, \mu\right)\right]}{\partial \mu^{n-1}} .
$$

Using Eq. 3 and Eq. 4, we have calculated the susceptibility ratios $\chi^{3} / \chi^{2}(\equiv S \sigma)$ and $\chi^{4} / \chi^{2}\left(\equiv \kappa \sigma^{2}\right)$ for net-baryon distribution. We have also estimated moments of net-proton multiplicity distributions using only primordial protons, anti-protons as well as the yields coming from the resonance decays. The resonance corrections are carried out using an average efficiency as discussed in Ref. [28]. We have noticed that within STAR acceptance, the differences between net-baryon and net-proton predictions are negligible. Therefore, in the present study we consider net-baryon distribution within STAR kinematic acceptance as used in Ref. [1. We parametrized the freezeout temperatures and chemical potentials using the relations, $\mu_{B}\left(\sqrt{s_{N N}}\right)=\frac{d}{1+e \sqrt{s_{N N}}}$ and $T\left(\mu_{B}\right)=a-b \mu_{B}^{2}-c \mu_{B}^{4}$. For most central collisions, the parameters are taken from Ref.[7, 29]. For peripheral collisions, we extracted these parameters from STAR preliminary data 30] for most peripheral $(70-80 \%)$ centrality collisions and the extracted parameters are given in Table 1. We use similar parametrization for $\mu_{S}$ and 
Net-baryon number fluctuations with the hadron resonance gas model using Tsallis distribution5

$\mu_{Q}$ as that of $\mu_{B}$ and the corresponding parameters are also listed in Table1. We set $\mu_{Q}$ to zero for peripheral collision as it does not have significant contribution as compared to $\mu_{B}$ and $\mu_{S}$.

Table 1. Parametrization of chemical potentials and freeze-out temperature extracted from the STAR experimental data for $(70-80) \%$ centrality 30 .

\begin{tabular}{llll}
\hline & $a(\mathrm{GeV})$ & $b\left(\mathrm{GeV}^{-1}\right)$ & $c\left(\mathrm{GeV}^{-3}\right)$ \\
\hline $\mathrm{T}$ & $0.158 \pm 0.002$ & $0.159 \pm 0.034$ & $0.500 \pm 0.001$ \\
\hline & $d(\mathrm{GeV})$ & $e\left(\mathrm{GeV}^{-1}\right)$ & \\
\hline$\mu_{B}$ & $0.900 \pm 0.059$ & $0.251 \pm 0.008$ & \\
$\mu_{S}$ & $0.239 \pm 0.001$ & $0.300 \pm 0.001$ & \\
\hline
\end{tabular}

In the context of heavy ion collision, the Tsallis distribution has a simple interpretation in terms of the distribution of temperatures (instead of a single Boltzmann temperature) of the fire ball which is created during the collision process [13. This can be shown mathematically as follows. The Tsallis-Boltzmann distribution as given in Eq. 2 can be written as,

$$
\left(1+\frac{(E-\mu)}{T_{f} k}\right)^{-k}=\int_{0}^{\infty} d\left(\frac{1}{T_{B}}\right) g\left(\frac{1}{T_{B}}, \frac{1}{T_{f}}\right) \exp \left(-\frac{(E-\mu)}{T_{B}}\right),
$$

where $k=1 /(q-1)$ and $g$ is the gamma function given by,

$$
g\left(\frac{1}{T_{B}}, \frac{1}{T_{f}}\right)=\frac{k T_{f}}{\Gamma(k)}\left(\frac{k T_{f}}{T_{B}}\right)^{k-1} \exp \left(-\frac{k T_{f}}{T_{B}}\right) .
$$

We assume a thermodynamic system constituting smaller systems which are described by a local temperature $T_{B}$, fluctuating from point to point around some equilibrium freeze-out temperature $T_{f}$. The inverse temperature of smaller systems, $\left(1 / T_{B}\right)$, is gamma distributed around a mean,

$$
\left\langle\frac{1}{T_{B}}\right\rangle=\frac{1}{T_{f}}
$$

and the parameter $q$ is related to the temperature fluctuation,

$$
\frac{\left\langle\left(\frac{1}{T_{B}}\right)^{2}\right\rangle-\left\langle\frac{1}{T_{B}}\right\rangle^{2}}{\left\langle\frac{1}{T_{B}}\right\rangle^{2}}=q-1,
$$

which becomes zero in the Boltzmann limit $(q \rightarrow 1)$. This leads to the interpretation of the Tsallis distribution at $T_{f}$ as a superposition of Boltzmann distributions with different $T_{B}$. The parameter $q$ describes the spread around the average temperature $T_{f}$. Finally, the average baryon density can be written as,

$$
n_{q}\left(T_{f}, \mu\right)=\int_{0}^{\infty} d\left(\frac{1}{T_{B}}\right) g\left(\frac{1}{T_{B}}, \frac{1}{T_{f}}\right) n^{B}\left(T_{B}\right) .
$$


Net-baryon number fluctuations with the hadron resonance gas model using Tsallis distribution6

where $n^{B}\left(T_{B}\right)$ is the average baryon density obtained with Boltzmann statistics. Using above relation, Eq. 4 can be expressed as weighted sum of the susceptibilities estimated using standard Boltzmann statistics and can be interpreted as an average taken over the whole phase space which is inhomogeneous in temperature. This is what will be measured experimentally if the temperature fluctuation exists in the hadronizing medium corresponding to a non-extensive value of $q$ which deviates from unity. Therefore, it is reasonable to argue that Eq. 4 can be used to estimate temperature averaged higher moments. It may be mentioned here that the above argument is strictly correct when quantum statistics is not important and Tsallis distribution can be written as the superposition of Boltzmann distributions. This condition is mostly true for baryons where quantum effects are small.
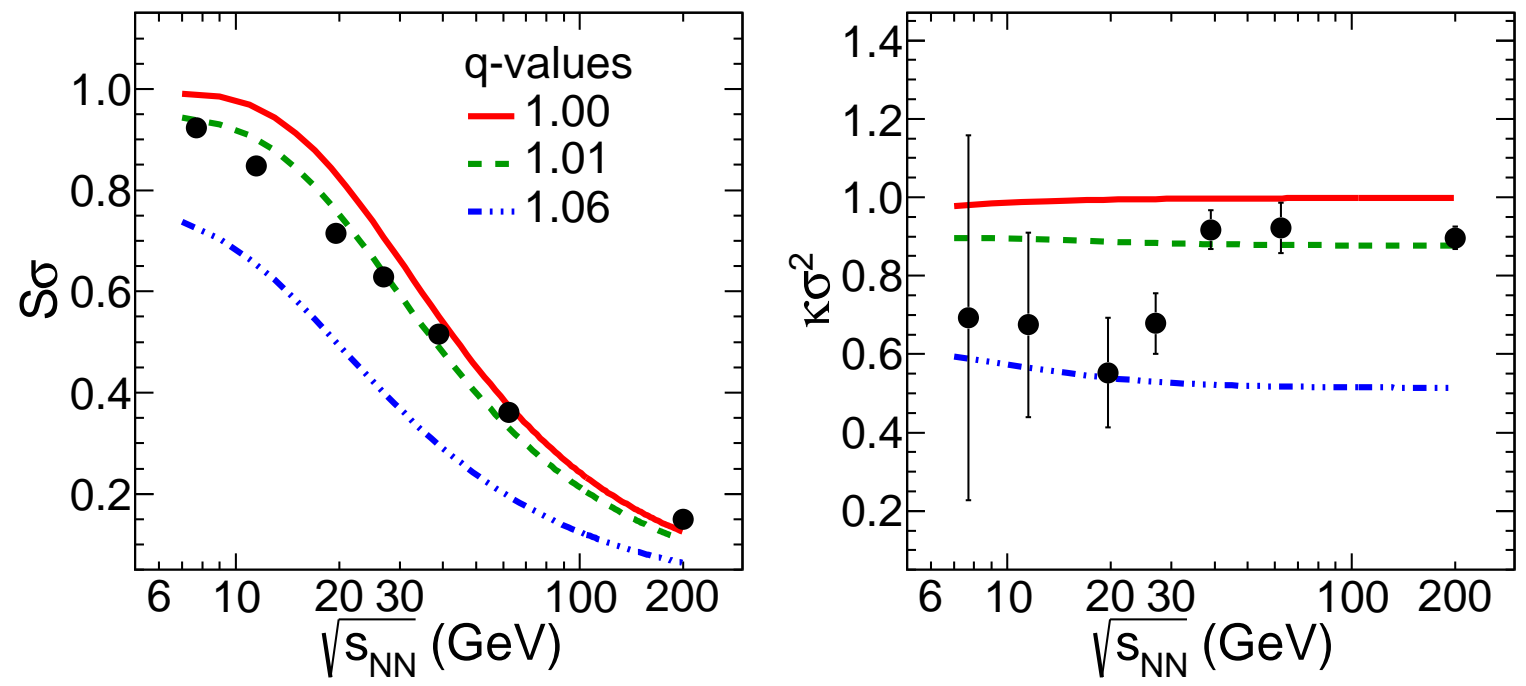

Figure 1. (Color online) The energy dependence of $S \sigma$ and $\kappa \sigma^{2}$ of net-baryon distribution calculated using HRG, where $q=1$ (solid curve), HRG-Tsallis with $q=1.01$ (dashed curve) and $q=1.06$ (dashed-dotted curve). A fixed $q$ value is used for all collision energies. The filled circles are STAR data for most central $(0-5 \%)$ in $\mathrm{Au}+\mathrm{Au}$ collisions [1].

Figure 1 shows the energy dependence of $S \sigma$ and $\kappa \sigma^{2}$ for central $(0-5 \%)$ $\mathrm{Au}+\mathrm{Au}$ collisions for STAR data [1]. Also shown are the predictions from HRG-Tsallis with different values of $q$. Higher value of $q$ indicates larger deviation from thermal equilibrium. For $q \rightarrow 1$, the $S \sigma$ and $\kappa \sigma^{2}$ values approach the HRG prediction [7]. Both $S \sigma$ and $\kappa \sigma^{2}$ decrease with increasing values of $q$ for all collision energies. With $q=1.01$, $S \sigma$ and $\kappa \sigma^{2}$ values from experimental data and HRG-Tsallis predictions are comparable at $\sqrt{s_{N N}}=39,62.4$ and $200 \mathrm{GeV}$. However, for the agreements between data and HRGTsallis predictions for $\kappa \sigma^{2}$ at 19.6 and $27 \mathrm{GeV}$, we require a higher value of $q=1.06$. The larger value of $q$ at these two energies would indicate the need of energy dependent parametrization of $q$. 
Net-baryon number fluctuations with the hadron resonance gas model using Tsallis distribution7

\section{Temperature dependent $q$ parameter}

The non-extensive $q$ parameter, which is generally extracted from the experimental data, assumes different values depending on the collision energy as well as on the centrality of the collisions. As discussed in Ref. [10, which uses transverse momentum spectra from the STAR experiment, the $q$ value decreases with increasing centrality indicating an evolution from a highly non-equilibrated system towards a more thermalized system. An increasing in centrality would mean higher temperature and lower baryochemical potential. At higher temperature, the system is expected to be closer to the equilibrium and gradually starts deviating from equilibrium as collision energy decreases or the collision becomes more peripheral. More specifically, $q$ should depend on both temperature $T_{f}$ and chemical potential $\mu_{B}$ at freeze-out. In this work, however, we adopt a simple approach and allow $q$ to depend only on $T_{f}$. On the other hand, $T_{f}$ is estimated from $\mu_{B}$ through a parametrization depending on the collision energy and the centrality of the collision as discussed before. Therefore, following Ref. [10], we express $q$ using the relation, $q=1+\left[\alpha\left(T_{0}-T_{f}\right)\right]^{1 / 2}$, where $T_{0}$ is a reference temperature close to the freeze-out temperature at $\sqrt{s_{N N}}=200 \mathrm{GeV}$ in central $(0-5 \%)$ collisions and is fixed at $0.167 \mathrm{GeV}$ such that $q=1$ for $T_{f} \geq T_{0}$.

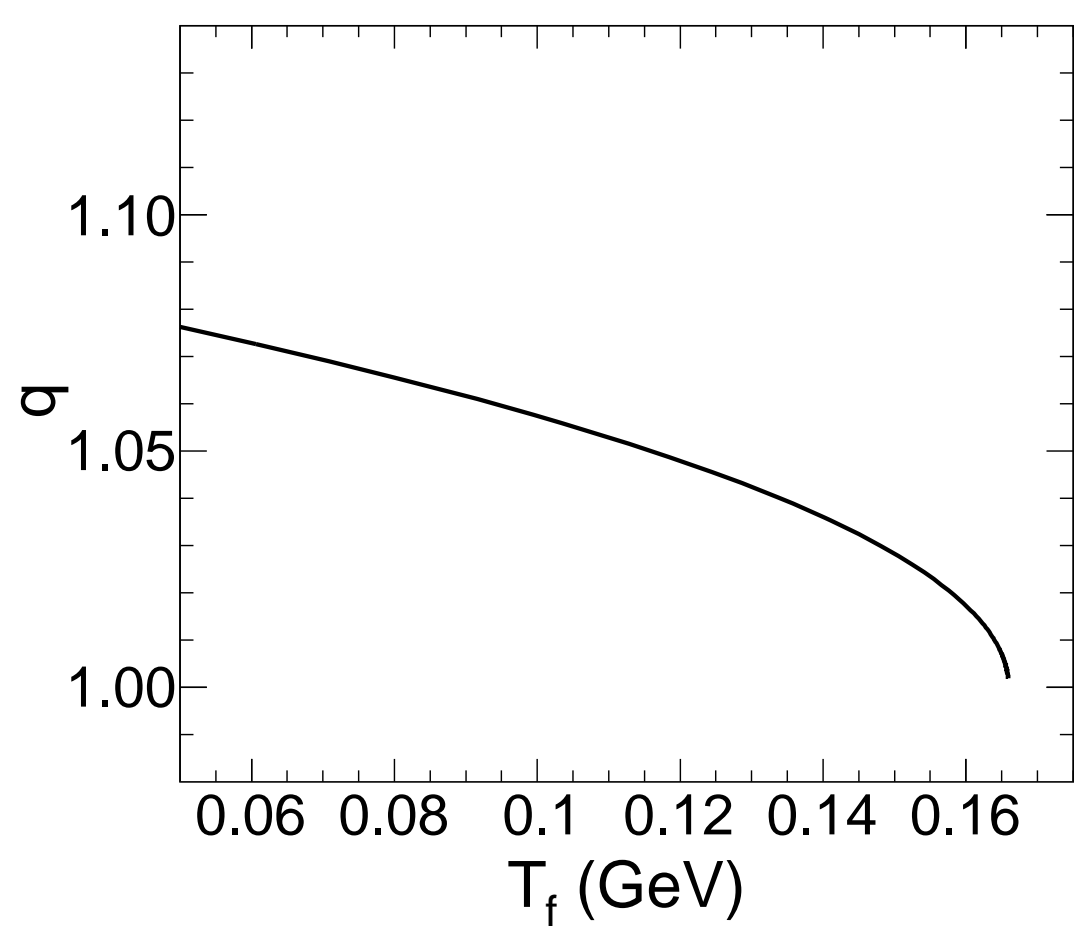

Figure 2. The non-extensive parameter $q$ as a function of temperature corresponding to different $\sqrt{s_{N N}}$ for $\alpha=0.05$.

Figure 2 shows the temperature dependence of non-extensive parameter $q$. The $q$-values increase with decreasing $T_{f}$, which would mean that, the deviation of the hadronizing system from the equilibrium increases as collision energy decreases or as the 
collision becomes more peripheral. The $q$ value approaches unity at $\sqrt{s_{N N}}=200 \mathrm{GeV}$ in central collisions. The parameter $\alpha$ is kept free and adjusted to fit the data. When $\alpha=0(q \rightarrow 1)$, we get back the HRG results. Note that we use the same parametrization irrespective of whether the collision is central or peripheral except different centrality which will have different chemical freeze-out parameters.

The partonic system, which is formed at high collision energy, has sufficient time between formation and freeze-out to achieve full equilibration, which corresponds to the region of $q=1$. This may not be the case at lower collision energies. The time available between formation and freeze out may not be sufficient to drive the system into the full equilibration. It could so happen that the hadronizing medium has attained only local equilibrium with different temperatures prevailing at different regions which can be considered as the fluctuations around some mean temperature $T_{f}$. This corresponds to the region of $q$ deviating from unity. However, by decreasing collision energy further, it may not be possible to produce the partonic system any more and the collisions may remain fully hadronic.
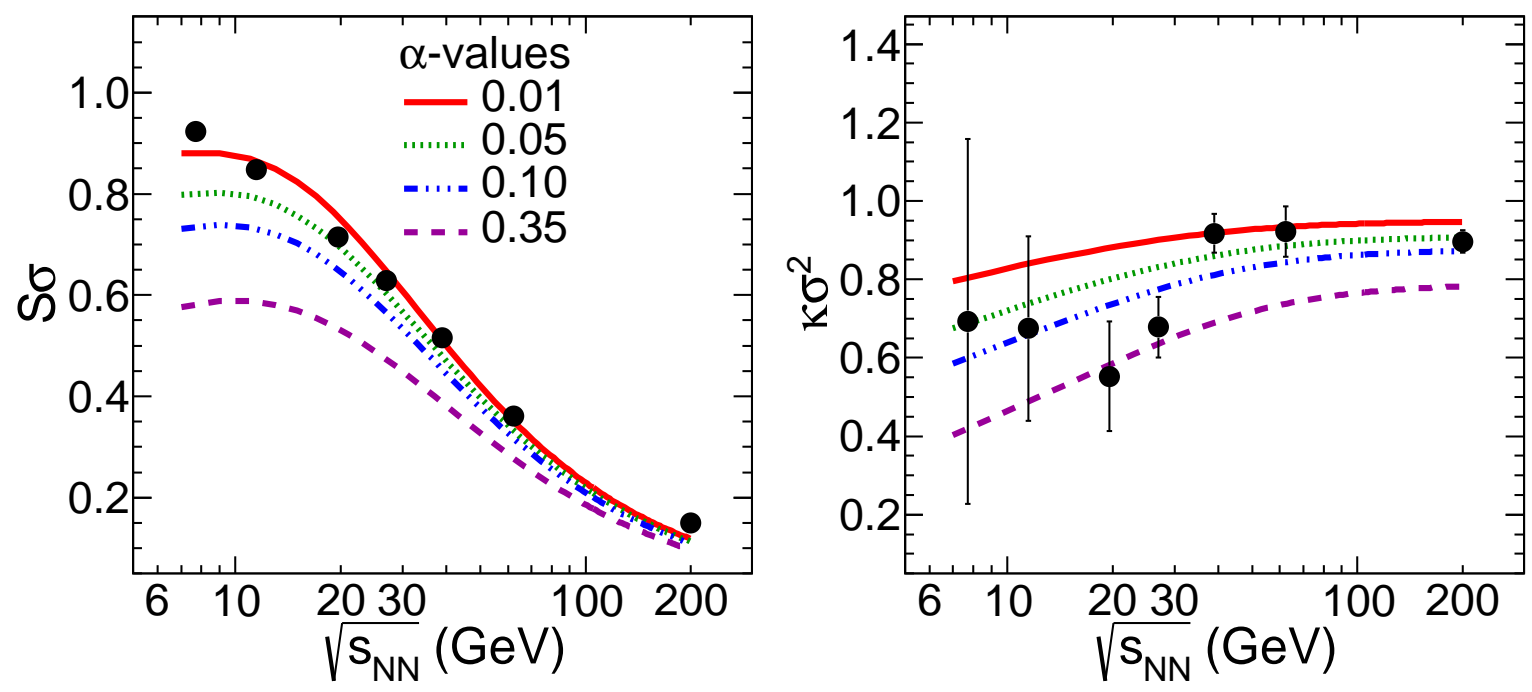

Figure 3. (Color online) The energy dependence of $S \sigma$ and $\kappa \sigma^{2}$ of net-baryon distribution calculated using HRG-Tsallis with different $\alpha$ values. The non-extensive parameter $q$ is related to $\alpha$ as described in the text varied with collision energy. The filled circles are STAR data for most central (0-5\% centrality) in Au+Au collisions [1].

\section{Results and Discussions}

Figure 3 shows the comparisons of $S \sigma$ and $\kappa \sigma^{2}$ between experimental data and HRGTsallis predictions with $q$ parametrization as shown in Fig. 2. For $S \sigma$ and $\kappa \sigma^{2}$ at $\sqrt{s_{N N}}=39,62.4$ and $200 \mathrm{GeV}$, HRG-Tsallis predictions with lower value of $\alpha=0.01$ (related to $q$ ), describes the experimental data very well. However, the $\kappa \sigma^{2}$ values at $\sqrt{s_{N N}}=19.6$ and $27 \mathrm{GeV}$ would require larger value of $\alpha(\sim 0.35)$ to match with 
the experimental data. This further emphasizes the strong energy dependence of nonextensive parameter $q$ at these particular energies, indicating larger deviations from the thermal equilibrium. We study the sensitivity of HRG-Tsallis results for $S \sigma$ and $\kappa \sigma^{2}$ by comparing the experimental data with model for peripheral $(70-80 \%)$ and central $(0-5 \%)$ collisions, using the $q$-parametrization.
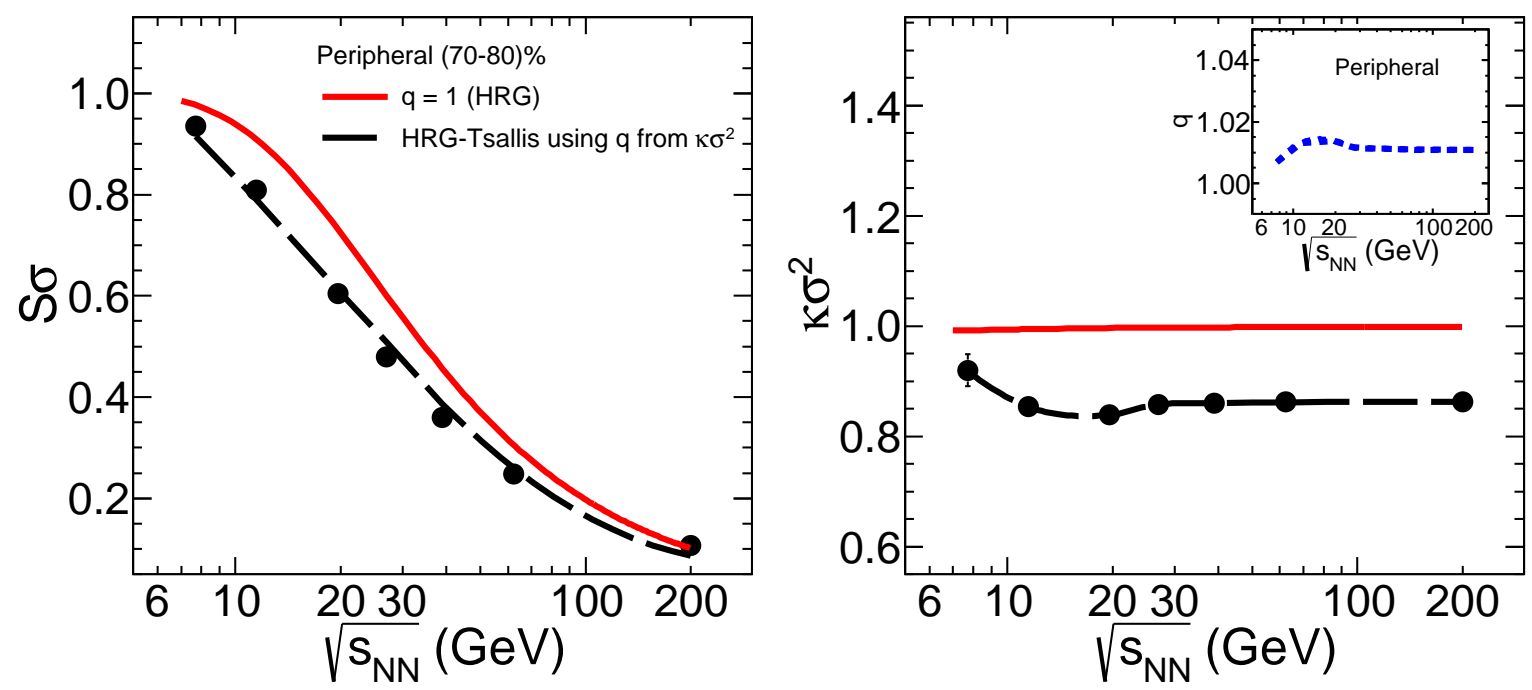

Figure 4. (Color online) The energy dependence of $S \sigma$ and $\kappa \sigma^{2}$ of net-baryon distribution calculated using HRG (solid curve) and HRG-Tsallis with different $q$ values. The filled circles are STAR data for most peripheral $(70-80 \%$ centrality) in $\mathrm{Au}+\mathrm{Au}$ collisions [1]. The inset in right panel shows the variation of $q$ as a function of collision energy.

Figure 4, shows the energy dependence of $S \sigma$ and $\kappa \sigma^{2}$ estimated using the freezeout parameters as listed in Table 1 for peripheral $(70-80 \%)$ collisions. As can be seen, the HRG results $(\alpha=0)$ show significant deviations from the experimental values [1], and $\kappa \sigma^{2}$ values in HRG model is always close to unity where the data points are about $15 \%$ below the HRG values. On the other hand, HRG-Tsallis with an average $q \sim 1.01$ can explain both $S \sigma$ and $\kappa \sigma^{2}$ very well for all the collision energies. The inset in Fig. [4 right panel shows the $q$ values used in HRG-Tsallis model to obtain a good agreement between experimental data of $\kappa \sigma^{2}$ and model results.

The corresponding results for most central $(0-5 \%)$ centrality collisions are shown in Fig. 5. The HRG predictions do not agree well the experimental data. We study the deviation of experimental data from the HRG results using two different methods. In first method, we reproduce the $S \sigma$ values by taking different $q$ values ranging between 1.0 to 1.015 in the HRG-Tsallis model. Figure 5 left panel shows the comparison of experimental data and the HRG-Tsallis results (dashed curve) for $S \sigma$ and $\kappa \sigma^{2}$ obtained from the first method. The experimental data for $S \sigma$ at all energies nicely agrees with the HRG-Tsallis with energy dependent $q$ values, but fails to explain the $\kappa \sigma^{2}$ values particularly at lower energies $\left(\sqrt{s_{N N}}<39 \mathrm{GeV}\right)$. In second method, we reproduce the 
Net-baryon number fluctuations with the hadron resonance gas model using Tsallis distribution10
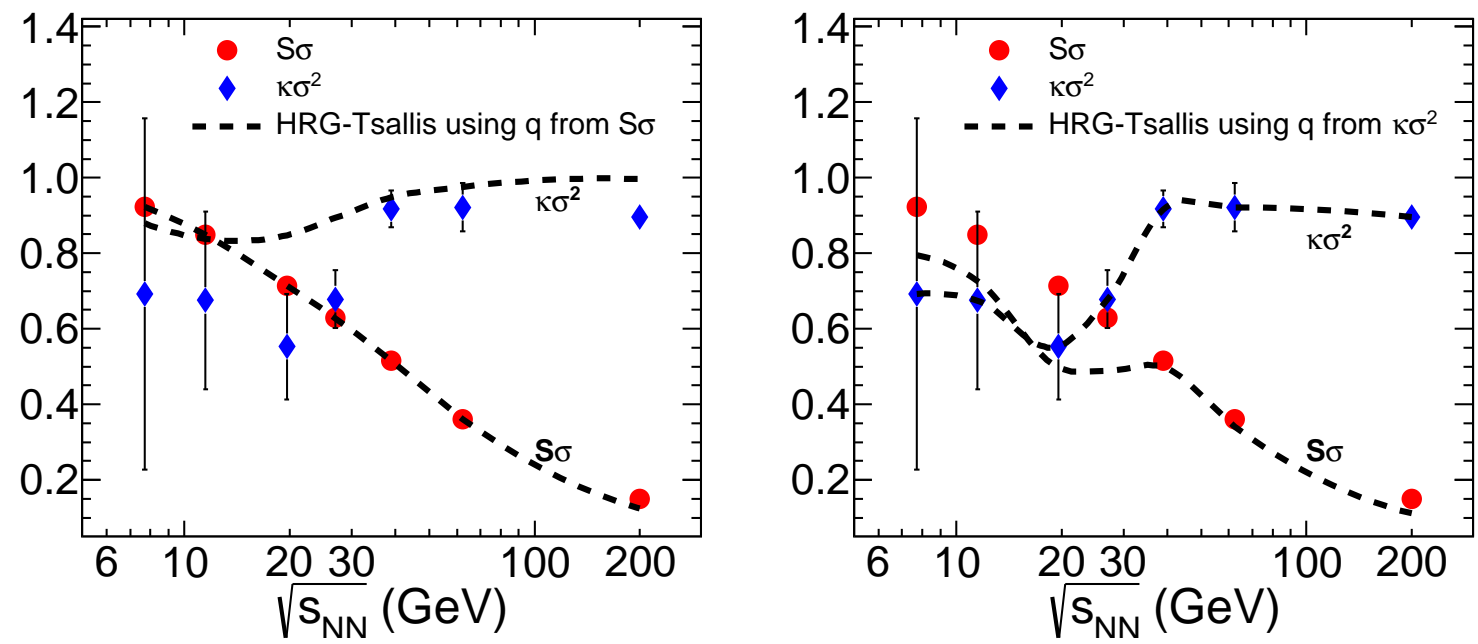

Figure 5. (Color online) The energy dependence of $S \sigma$ and $\kappa \sigma^{2}$ of net-baryon distribution calculated using HRG-Tsallis with different $q$ values. Left panel shows the HRG-Tsallis calculations which reproduce the experimental $S \sigma$ data and right panel shows the HRG-Tsallis calculations which reproduce the experimental $\kappa \sigma^{2}$ data. The symbols are STAR data for most central ( $0-5 \%$ centrality) in Au+Au collisions [1.

experimentally measured $\kappa \sigma^{2}$ data using different $q$ values ranging from 1.0 to 1.06 in the HRG-Tsallis model. Figure 5 right panel shows the comparison of experimental data and the HRG-Tsallis results (dashed curve) for $S \sigma$ and $\kappa \sigma^{2}$ obtained from the second method. Although using higher values of $q$ can explain the energy dependence of $\kappa \sigma^{2}$ at lower energies, but fails to explain the $S \sigma$ experimental data as shown in the right panel of Fig. 5. The agreements between experimental data and HRG-Tsallis model for $S \sigma$ and $\kappa \sigma^{2}$, using a variable $q$ values, are difficult to obtain simultaneously under the current framework. The energy dependence of $q$ for $(0-5 \%)$ central collisions are shown in Fig. 6 for the above discussed methods. For central collisions, using $q$ values obtained from reasonable agreement between HRG-Tsallis and experimental data for $S \sigma$, there is smooth increase of $q$ ranging from 1.0 to 1.015 as we go from higher energies to lower energies. In second method, where we obtain $q$ values from reasonable agreement between HRG-Tsallis and the experimental measured $\kappa \sigma^{2}$ values, there is a sudden increase of $q$ parameter from 1.0 to 1.06 for energies below $39 \mathrm{GeV}$. Higher values of $q$ indicates larger deviation of the system from thermal equilibrium. Similar observation is also reported in Ref. [31.

It may be mentioned here that, we have not considered the Van der Waals (VDW) type excluded volume effect which could be an another source of deviation of $S \sigma$ and $\kappa \sigma^{2}$ from the HRG predictions [32]. However, the VDW type deviation increases with decreasing energies where as the STAR experimental values have maximum deviation at $\sqrt{s_{N N}}=19.6 \mathrm{GeV}$ and less deviation at the two lower RHIC energies with larger uncertainties. Therefore, we have not considered VDW type effect in the 
Net-baryon number fluctuations with the hadron resonance gas model using Tsallis distribution11

present calculation. The transport model like UrQMD within STAR acceptance also produces similar results for $S \sigma$ and $\kappa \sigma^{2}$ which decrease with decreasing energies. Tsallis distribution also includes quantum effect which is important at lower collision energies. Therefore, in this work we explore, how much non-statistical fluctuations inherently present in the Tsallis non-extensive distribution which can explain the experimental observations without considering other dynamical effects.

Another aspect which has not been considered in current HRG-Tsallis model is the effect of non-extensivity on the freeze-out parameters which are generally extracted from the experiments using HRG model in grand canonical ensemble. It is observed in Ref. 31] that while freeze-out temperature decreases, chemical potential increases with increasing $q$ parameter. However, for $q<1.01$ which has been used in the present study, we notice that the increase in $\mu_{B}$ is not significant although $T_{f}$ decreases by about $10 \%$ from the value when $q=1$. As argued in [31, since Tsallis distribution is broader than the Boltzmann distribution, temperature needs to be decreased in order to conserve the particle density. Therefore, we have estimated the moments keeping $\mu_{B}$ unchanged but allowing freeze-out temperature to decrease up to $10 \%$. Interestingly, we notice that while $S \sigma$ increases slightly, $k \sigma^{2}$ remains unchanged. This suggests that $S \sigma$ is sensitive to both temperature and chemical potential while $\kappa \sigma^{2}$ is more sensitive to $q$ parameter.

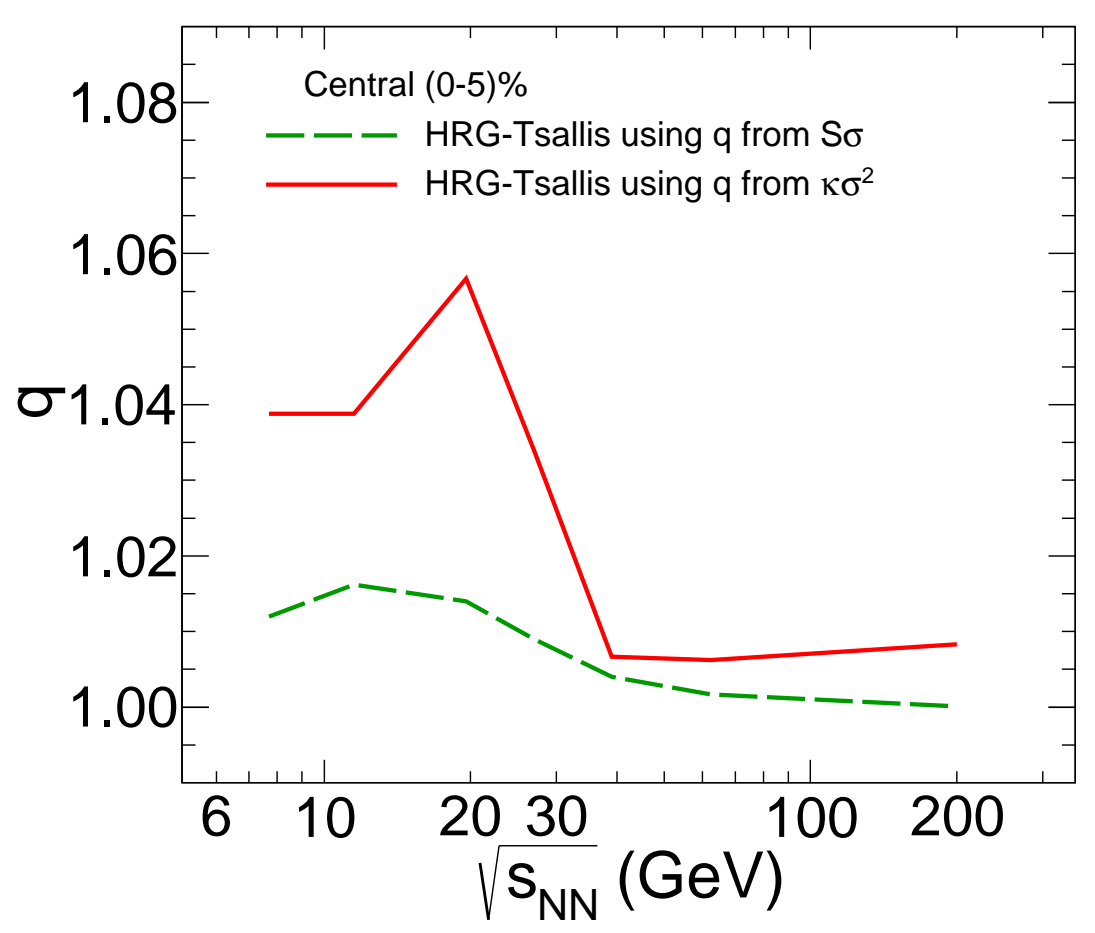

Figure 6. (Color online) The energy dependence of non-extensive parameter $q$ using HRG-Tsallis for central $(0-5 \%)$ collisions. The dashed curve shows the $q$ values obtained from reasonable agreement of $S \sigma$ between experimental and HRG-Tsallis results. The solid curve shows the $q$ values obtained from reasonable agreement of $\kappa \sigma^{2}$ between experimental and HRG-Tsallis results. 
Net-baryon number fluctuations with the hadron resonance gas model using Tsallis distribution12

\section{Summary}

In conclusion, we have studied the energy dependence of the fluctuations of net-baryon productions through higher moments namely $S \sigma$ and $\kappa \sigma^{2}$ using HRG with Tsallis nonextensive distribution function. When the non-extensive parameter $q$ is close to unity, the moments obtained using HRG-Tsallis model can be interpreted as the weighted average of the moments estimated using many Boltzmann distributions corresponding to the distribution of temperatures over the whole phase space. It is shown that HRGTsallis model can explain the energy dependence of $S \sigma$ and $\kappa \sigma^{2}$ measurements of the most peripheral collisions which is otherwise difficult to explain using the normal HRG model which predicts $\kappa \sigma^{2}$ close to unity for net-baryon productions. The HRG-Tsallis model also explains the energy dependence of $S \sigma$ data of central collision. However, the model can not explain the corresponding $k \sigma^{2}$ values for the central collisions particularly at energies $19.6 \mathrm{GeV}$ and $27 \mathrm{GeV}$ at the same time. This deviation is so significant that it is an indication of the presence of additional fluctuations at around $20 \mathrm{GeV}$ which may have some dynamical origin not contained in the HRG-Tsallis model. These dynamical fluctuations may originate from a QCD phase transition of hadronic to partonic medium. This study also provides an alternative baseline for the experimental results for the moments of the net-proton multiplicity distributions which may indicate the possible presence of a phase transition and the critical point in high energy heavy-ion collisions.

\section{Acknowledgments}

Financial assistance from the Department of Atomic Energy, Government of India is gratefully acknowledged. PG acknowledges the financial support from CSIR, New Delhi, India.

[1] L. Adamczyk et al. [STAR Collaboration], Phys. Rev. Lett. 112 (2014) 032302 arXiv:1309.5681 [nucl-ex].

[2] A. Bazavov, H. T. Ding, P. Hegde, O. Kaczmarek, F. Karsch, E. Laermann, S. Mukherjee and P. Petreczky et al., Phys. Rev. Lett. 109 (2012) 192302 [arXiv:1208.1220 [hep-lat]].

[3] M. M. Aggarwal et al. [STAR Collaboration], Phys. Rev. Lett. 105 (2010) 022302 arXiv:1004.4959 [nucl-ex]].

[4] S. Gupta, X. Luo, B. Mohanty, H. G. Ritter and N. Xu, Science 332 (2011) 1525 arXiv:1105.3934 [hep-ph]].

[5] The Skellam distribution is the discrete probability distribution of the difference $N_{1}-N_{2}$ where $N_{1}$ and $N_{2}$ are two random variables each follwing Poisson distribution.

[6] A. Andronic, P. Braun-Munzinger and J. Stachel, Nucl. Phys. A 772 (2006) 167 nucl-th/0511071].

[7] F. Karsch and K. Redlich, Phys. Lett. B 695 (2011) 136 [arXiv:1007.2581 [hep-ph]].

[8] M. A. Stephanov, Phys. Rev. Lett. 107 (2011) 052301 arXiv:1104.1627 [hep-ph]].

[9] M. Cheng, P. Hendge, C. Jung, F. Karsch, O. Kaczmarek, E. Laermann, R. D. Mawhinney and C. Miao et al., Phys. Rev. D 79 (2009) 074505 arXiv:0811.1006 [hep-lat]].

[10] Z. Tang, Y. Xu, L. Ruan, G. van Buren, F. Wang and Z. Xu, Phys. Rev. C 79 (2009) 051901 arXiv:0812.1609 [nucl-ex]].

[11] C. -Y. Wong and G. Wilk, Acta Phys. Polon. B 43 (2012) 2047 arXiv:1210.3661 [hep-ph]].

[12] J. Cleymans and D. Worku, J. Phys. G 39 (2012) 025006 arXiv:1110.5526 [hep-ph]].

[13] G. Wilk and Z. Wlodarczyk, Phys. Rev. Lett. 84 (2000) 2770 hep-ph/9908459;

Phys. Rev. C 79 (2009) 054903 arXiv:0902.3922 [hep-ph]]. 
Net-baryon number fluctuations with the hadron resonance gas model using Tsallis distribution13

[14] G. Wilk and Z. Wlodarczyk, collisions," Eur. Phys. J. A 48 (2012) 161 arXiv:1203.4452 [hep-ph]].

[15] L. Stodolsky, Phys. Rev. Lett. 75 (1995) 1044.

[16] C. Tsallis, Introduction to Nonextensive Statistical Mechanics, Springer (2009); J. Stat. Phys. 52 (1988) 479.

[17] G. Wilk and Z. Wlodarczyk, Physica A 376 (2007) 279 cond-mat/0603157 [cond-mat.stat-mech]].

[18] B. Berdnikov and K. Rajagopal, Phys. Rev. D 61, 105017 (2000) hep-ph/9912274].

[19] M. A. Stephanov, Phys. Rev. Lett. 102 (2009) 032301 [arXiv:0809.3450 [hep-ph]].

[20] A. Robledo, Phys. Rev. Lett. 83 (1999) 2289.

[21] A. Adare et al. [PHENIX Collaboration], Phys. Rev. D 83 (2011) 052004 arXiv:1005.3674 [hepex]].

[22] B. I. Abelev et al. [STAR Collaboration], Phys. Rev. C 75 (2007) 064901 nucl-ex/0607033.

[23] KAamodt et al. [ALICE Collaboration], Phys. Lett. B 693 (2010) 53 arXiv:1007.0719 [hep-ex]].

[24] V. Khachatryan et al. [CMS Collaboration], JHEP 1002 (2010) 041 arXiv:1002.0621 [hep-ex]].

[25] B. -C. Li, Y. -Z. Wang, F. -H. Liu, X. -J. Wen and Y. -E. Dong, Phys. Rev. D 89 (2014) 054014 arXiv:1403.4025 [hep-ph]].

[26] G. Wilk and Z. Wlodarczyk, Eur. Phys. J. A 40 (2009) 299 arXiv:0810.2939 [hep-ph]].

[27] J. Cleymans and D. Worku, Eur. Phys. J. A 48 (2012) 160 arXiv:1203.4343 [hep-ph]].

[28] P. Garg, D. K. Mishra, P. K. Netrakanti, B. Mohanty, A. K. Mohanty, B. K. Singh and N. Xu, Phys. Lett. B 726 (2013) 691 [arXiv:1304.7133 [nucl-ex]].

[29] J. Cleymans, H. Oeschler, K. Redlich and S. Wheaton, Phys. Rev. C 73 (2006) 034905 hep-ph/0511094.

[30] S. Das [STAR Collaboration], Nucl. Phys. A904-905 2013 (2013) 891c arXiv:1210.6099 [nucl-ex]].

[31] J. Cleymans, G. Hamar, P. Levai and S. Wheaton, J. Phys. G 36 (2009) 064018 arXiv:0812.1471 [hep-ph]].

[32] J. Fu, Phys. Lett. B 722 (2013) 144. 